\title{
Celebrating partnership working between the third sector and the NHS
}

\author{
Emeritus Professor Alan Glasper, University of Southampton, discusses the success of partnerships between \\ the third sector and the NHS which is crucial to improving care for people in society.
}

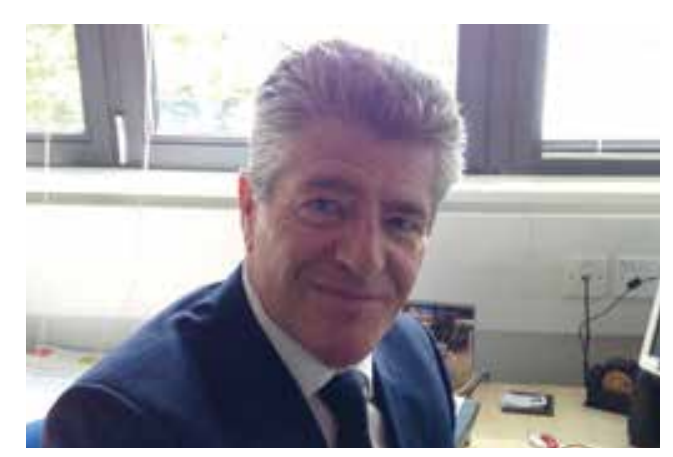

he third sector of health and social care embraces those charities and other organisations who make significant contributions to the overall success of the NHS in providing care for discrete patient groups. NHS England actively encourages these organisations' input into the overall structure of the health service (NHS England, 2019). Third-sector charities, ie organisations not in the public or private domain, are often the unsung heroes of healthcare provision. Could we imagine for example cancer care without Macmillan Cancer Support or Marie Curie services?

It would be impossible to cover the contributions of all the organisations that contribute to the NHS, but it is timely to look at one particular charity that is celebrating 40 years of service to the NHS: this is Bliss, a charity for babies born premature or sick.

\section{Background}

Bliss was launched in 1979, initially to raise money for equipment such as incubators for premature babies and those requiring care in neonatal units. The charity has since provided much more than equipment - it exists to ensure that babies requiring neonatal care in units across the UK have the best chance of survival and quality of life.

It was founded by a group of concerned parents who realised that hospitals with neonatal units did not have access to all the equipment or the trained staff required to provide the babies with optimum care. Forty years on the charity continues to work with neonatal units to enhance the care these vulnerable babies need not only to survive but to thrive with none or minimum disability.

A seminar held at the Welcome Institute in 1999, entitled Origins of Neonatal Intensive Care in the UK (Christie and Tansey, 2001), provided a fascinating collection of witness statements from doctors and nurses who had been at the forefront of the development of neonatology as a discrete discipline. This revealed that it was in January 1946 that the first premature baby unit opened with eight cots at Southmead Hospital in Bristol.

It was a troublesome time for those pioneers in neonatal care because there were no plastic or disposable materials in the immediate postwar years, no incubators were available and no piped oxygen. Crucially, there were no specially trained neonatal medical or nursing staff. One of the first neonatal units with a specific research interest in respiratory lung disease opened at University College Hospital London in 1954 with 12 cots. The staff quickly became aware of the high incidence of subsequent disability among premature neonatal survivors.

Even by 1978 there were only 12 paediatricians in England and Wales who worked predominantly with neonates. However, many nurses were beginning to specialise in caring for sick premature babies and one in particular, the late Miss Anthea Blake who had a background in midwifery and paediatric nursing, joined the neonatal nursing team at University College Hospital London in 1968. Anthea was one of the six founder members of the Neonatal Nurses' Association and played a large part in helping the new charity Bliss to thrive following its launch. Since then, charities such as Bliss have helped bridge the funding gap in neonatal care.

\section{The Bliss strategy 2019-2022}

The mandate of the charity is to ensure that each premature or sick baby, irrespective of where he or she is born is given optimum care and treatment to ensure survival and, importantly, a good quality of life that is as free from disability as possible.

The achievement of best outcomes for these babies does not come cheaply and requires considerable resources. The equipment and care environment is partially provided by charities such as Bliss who also provide cadres of neonatal volunteers. One of the charity's key achievements was the launch of its Baby Charter in 2009 (Campbell and Chivers, 2011; Bliss, 2019a), which helps neonatal units across the UK to introduce family-centred care standards through an accreditation process. These are pertinent to many areas of neonatal care, for example:

- The emotional support available to families

- How parents are consulted in decisions regarding their baby

- The facilities a neonatal unit has to enable parents to be involved in their baby's care, such as overnight accommodation

This Bliss Charter gives recommendations and support for neonatal units to introduce lasting improvements that ensure families are consistently involved in care. The Bliss strategy report shows that in excess of $89 \%$ of neonatal units across the country have adopted the charter. The strategy has five objectives, which aim to ensure that babies born sick or premature (Bliss, 2019b):

- Have the best chance of survival

- Have well-supported parents playing an active role in decision-making and care

- Are cared for in neonatal environments that best support babies' development

- Benefit from neonatal research that can lead to practical improvements in their care

- Receive high-quality care within a neonatal health system that is appropriately funded, structured and staffed.

\section{Best chance of survival}

The charity's primary goal is to ensure more babies born sick or premature survive. This is important, because UK neonatal mortality rates 
lag behind those of similar countries in Europe. In 1990, the UK was seventh in the European Union for neonatal deaths, but by 2015 it had fallen to 19th position, behind countries such as Germany, Sweden, and France (Office for National Statistics, 2017).

Bliss works in partnership with similar charities to lobby the Government to ensure it delivers the policy changes necessary to meet the target of reducing neonatal and stillborn deaths by $50 \%$ by 2025 . Furthermore, the charity wants to ensure that lessons learnt from every neonatal death are shared and cascaded at national level. The Government is being proactive and in March it launched a stillbirth and infant mortality tool to model estimates of the possible effects of various factors on infant mortality and stillbirths in a specific geographical locality (Public Health England, 2019).

The neonatal database BadgerNet (Clevermed, 2017), which is used throughout the UK and further afield, is a useful vehicle for research and benchmarking; for example, Heathcote et al (2018) used this database to examine the timing and documentation of key events in neonatal resuscitation.

Well-supported parents playing active role The bonding process is critical in the early days of life and since its launch Bliss has campaigned for parents to be given a greater hands-on role in their baby's care. Bliss staff understand how difficult this can be within the confines of the neonatal care environment.

Flacking et al (2012) discussed the importance and impact of physical and emotional closeness between the pre-term infant and parent in the neonatal intensive care unit. Hence, the new Bliss strategy seeks to develop better information for parents and provide them with better emotional support through its extensive volunteer network. Bliss also wants to continue promoting its charter throughout UK neonatal units and resolve barriers to providing family-centred care within individual units. The charity is especially interested in enhancing accommodation and facilities for parents on neonatal units, such as kitchens and rest rooms.

\section{Care that best supports development}

Bliss considers that developmental and familycentred approaches to care benefit both babies born premature or sick and their families. The charity offers and takes a leading role in training health professionals on the importance of this aspect of care. Bliss intends to continue funding research that expands the evidence base on how best to deliver developmental care and improve outcomes.

\section{Benefit from neonatal research}

Achieving the best outcomes for babies born prematurely or sick lies at the centre of what Bliss does. By raising money to fund and support research, the charity hopes to continue investing in children's health and wellbeing through continuous improvement of their care.

Bliss focuses on supporting research that explores the parents' perspectives. For example, it is currently funding work by Alison McFadden, who is both a midwife and a Professor of Mother and Infant Public Health at the University of Dundee, to investigate how to optimally help parents and staff in neonatal units to feed premature babies (https://tinyurl. com/bliss-research).

\section{High-quality and well-resourced care}

Bliss aims to ensure that babies born prematurely or sick receive high-quality care in a neonatal health system that is appropriately funded, structured and staffed. Technological advances now allow babies who in the past would have died to live and grow, meaning that demand for neonatal services is unlikely to ease.

Growing demand also raises questions about the sustainability of the neonatal workforce, .especially neonatal intensive care nurses and advanced neonatal nurse practitioners. Nurses wishing to qualify in a specialty require further training. Such specialist education once came under the auspices of the national boards for nursing in each of the four countries of the UK. Since the dissolution of the national boards and because the Nursing and Midwifery Council no longer specifies post-qualifying course curricula there have been concerns that there might be loss of parity between universities.

Furthermore pressures on post-qualifying training funding mean nurses are finding it more difficult to access appropriate neonatal courses. Like Bliss, the British Association of Perinatal Medicine (2010) is concerned about the future provision of neonatal nurses with the appropriate skill set. Bliss will continue to lobby for the resourcing of appropriate staffing of neonatal units by nurses or midwives who have developed the skills, knowledge and attitudes necessary. Bliss is well aware of the importance of helping ensure that additional resources are invested to build the neonatal workforce needed for the future.

\section{KEY POINTS}

- Third-sector charities make a significant contribution to the overall success of the NHS in providing care for discrete patient groups

- Neonatal charity Bliss is celebrating 40 years of service to the NHS in enhancing the care of premature and sick babies

- The mandate of Bliss is to ensure premature or sick babies, irrespective of where they are born, are given optimum care and treatment to ensure survival and a good quality of life

- The new Bliss strategy has five objectives, principally to ensure that a larger percentage of babies born sick or premature survive

\section{Conclusion}

Bliss is but one of many charities that help sustain the NHS, especially during periods of economic downturn. This article has celebrated the contribution of the many unsung heroes who help keep the NHS functioning at optimum level. It is important to stress that many nurses across the country work actively with charities such as Bliss to help them achieve their goals such as endeavouring to give every baby born prematurely or sick the very best start on life's journey. BJN

Bliss. What's involved in the Bliss Baby Charter? 2019a. https://tinyurl.com/y3smbk8d (accessed 3 June 2019) Bliss. Our strategy. 2019b. https://tinyurl.com/y5tr2f8j (accessed 3 June 2019)

British Association of Perinatal Medicine. Service standards for hospitals providing neonatal care. 3rd edn. 2010. https://tinyurl.com/y6xlxhef (accessed 3 June 2019)

Campbell C, Chivers Z. Bliss self-assessment audit tool pilot. Infant 2011; 7(6): 176-178. https://tinyurl.com/ y5g9t5ct (accessed 4 June 2019)

Christie DA, Tansey EM. Origins of neonatal intensive care in the UK. 2001. https://tinyurl.com/y6k7deyc (accessed 3 June 2019)

CleverMed. BadgerNet Neonatal-EPR. 2017. https:// tinyurl.com/yxckkkna (accessed 3 June 2019)

Flacking R, Lehtonen L, Thomson G et al. Closeness and separation in neonatal intensive care. Acta Paediatr. 2012; 101(10): 1032-1037. https://doi.org/ 10.1111/j.1651-2227.2012.02787.x

Heathcote AC, Jones J, Clarke P. Timing and documentation of key events in neonatal resuscitation. Eur JPediatr 2018; 177(7): 1053-1056. https://doi.org/ 10.1007/s00431-018-3160-8

NHS England.Voluntary sector partnerships. 2019. https:// tinyurl.com/y5nker6a (accessed 3 June 2019)

Office for National Statistics. UK drops in European child mortality rankings. 2017. https://tinyurl.com/yyl4mu2p (accessed 3 June 2019)

Public Health England. Pregnancy and early life: reducing stillbirth and infant death. 2019. https://tinyurl.com/ y5dp9u5z (accessed 3 June 2019) 